\title{
Tandem mass spectrometry-based identification of protein profiles in the cerebrospinal fluid of Alzheimer's dementia patients
}

Anamika Misra ( $\sim$ anamikamsr14@gmail.com )

Banaras Hindu University Institute of Medical Sciences https://orcid.org/0000-0003-3551-0011

Sankha Shubhra Chakrabarti

Banaras Hindu University Institute of Medical Sciences

Indrajeet Singh Gambhir

Banaras Hindu University Institute of Medical Sciences

Meghraj Singh Baghel

Johns Hopkins University

Yugendra Ramchandra Patil

National Chemical Laboratory CSIR

Research Article

Keywords: Alzheimer's disease, Biomarker, CSF, Proteomics, LC-MS/MS

Posted Date: June 3rd, 2021

DOl: https://doi.org/10.21203/rs.3.rs-574644/v1

License: (9) This work is licensed under a Creative Commons Attribution 4.0 International License.

Read Full License 


\section{Abstract}

Background: Alzheimer's disease (AD) is the most common form of dementia and about two thirds cases are diagnosed late due to a long asymptomatic phase. There exists the need for newer biomarkers which can add accuracy to $A D$ diagnosis, detect $A D$ at an early stage, as also lend new pathogenic insights into $A D$. Recent AD biomarker discovery has focused on proteomic approaches, especially in the cerebrospinal fluid.

Methods: We used a bottom-up proteomic approach. Cerebrospinal fluid (CSF) samples from 6 patients with $A D$ and 6 controls were digested with trypsin and analyzed by using LC-MS/MS (tandem mass spectrometry). The peptide data from CSF samples of both AD and control groups was then subjected to bioinformatics analysis with STRING version 11.0. Protein-protein interaction networks were constructed, and enrichment analysis performed.

Results: Significant up-regulation of 13 proteins in the CSF was observed in AD cases in comparison to controls, while 30 proteins were down-regulated. APOE and LGALS3BP were the upregulated proteins involved in closed network and the downregulated proteins were F2, PENK, IGF2, APOH, SAA1, AHSG, SPP1 and CD44. APOE, APOH, F2 and PENK shared common involvement in multiple biological processes as evident on enrichment. Regulation of insulin like growth factor involving IGF2, F2, APOE and AHSG and glycosaminoglycan binding involving APOE, APOH, F2, SAA1, and CD44 were major pathways of interest determined on bioinformatic analysis.

Conclusion: Our study identified novel tentative biomarkers of AD which included F2, PENK and SAA1, as well as reinforced earlier described biomarkers such as APOE and AHSG. These findings need to be validated in larger sample sizes to evaluate their utility as true biomarkers. Further, the pathways of interest- insulin like growth factor regulation and glycosaminoglycan binding need to be studied further in the context of $A D$.

\section{Introduction}

Alzheimer's disease (AD) is the most common cause of dementia in the elderly. ${ }^{1}$ AD accounts for more than half of the cases of all-cause dementia in those $>60$ years of age. In India, the incidence of $A D$ is 4.7 to 5.34 per 1000 person-years. ${ }^{2}$ Late onset $A D$ accounts for $90 \%$ of all $A D$ cases. ${ }^{3} A D$ is characterized by an early loss of episodic memory, and a gradually progressive course over years finally resulting in a patient who has lost most of their cognitive functions and is entirely dependent on caregiver support.

There is no single confirmatory test for AD diagnosis and about two-thirds of dementia cases may be undiagnosed due to the long duration of an asymptomatic phase, during which pathophysiological mechanisms are building up. ${ }^{3}$ Revised guidelines for diagnosing $A D$ were proposed by the National Institute of Aging and Alzheimer's work group (published in 2011). They recommend that AD should be considered a slowly progressive brain disease that begins well before clinical symptoms emerge, and lay 
new-found emphasis on biomarkers to complement the clinical diagnosis of AD. ${ }^{4}$ Both the Committee for Medicinal Products for Human Use of the European Medicines Agency (EMA) and the US Food and Drug Administration (FDA) prioritize the use of biomarkers. ${ }^{5}$ Biomarkers are not only important for AD diagnosis because of their utility in picking up early disease, but also because the pathogenesis of $A D$ is not entirely clear till now. The amyloid $\beta$ plaque and tau-based neurofibrillary tangle hypothesis, although most widely accepted, have their share of dissidents. Several other mechanisms such as oxidative stress, mitochondrial dysfunction, brain-limited metabolic syndrome, abnormalities of cholesterol metabolism have been highlighted. ${ }^{6}$ Hence, novel biomarkers may aid in charting the AD pathogenetic pathway.

Among the platforms developed to carry out research in biomarkers for neurodegenerative diseases, with $A D$ being studied most extensively, proteomics has gathered high consideration. ${ }^{7}$ Neuroproteomics is a systems biology-based approach that involves the large-scale profiling and functional annotation of brain proteins. ${ }^{8}$ A particularly attractive technique of neuroproteomics is shotgun proteomics using liquid chromatography-tandem mass spectrometry (LC-MS/MS). The cerebrospinal fluid (CSF) is an ideal sample for such investigations, providing a focused window to studying neurodegeneration. Studies have reported several novel CSF proteins as unique biomarkers for early detection of Alzheimer's disease. $9,10,11,12,13$ However, literature concerning Indian patients with AD is scarce. A single study from NIMHANS from southern India reported differential expression of the proteins MAPT, NPTX2, VGF, GFAP, NCAM1 in the CSF of AD patients as compared to non-AD controls. This study utilized high-resolution mass spectrometry. ${ }^{14}$ Our study was designed to identify tentative CSF protein biomarkers of AD by LCMS/MS (liquid chromatography-tandem mass spectrometry). We also planned a bioinformatics analysis of the data obtained using STRING to try to identify potential pathogenetic clues for AD by studying protein-protein interaction (PPI) networks.

\section{Materials And Methods}

2.1 Study design and Sample size: This was a single center, case-control study with a case group (probable AD patients) and an age-sex matched control group (cognitively normal). Based on some previous studies, 6 CSF samples from AD patients and 6 control CSF samples were planned to be collected.

2.2 Sample collection, storage, and processing: After obtaining written informed consent, $2 \mathrm{ml}$ of CSF was collected from all patients enrolled in the study in sterile containers and was stored at $-80^{0} 1 \mathrm{ml}$ of CSF sample was similarly collected from each cognitively normal subject after written informed consent and was also stored at $-80^{\circ} \mathrm{C}$.

2.3 Study setting and participants: The study was conducted between June 2013 and May 2016 in a large tertiary medical university hospital in north India. AD patients were enrolled from the out-patients' clinic of the department of Geriatric Medicine by random selection. Patients more than 60 years of age suspected to have dementia based on initial history were evaluated for making a diagnosis of probable 
AD. Written informed consent was taken from each participant or the legal guardian, as was deemed suitable. The patients of probable AD $(n=6)$ were diagnosed using the DSM- 5 criteria after initial screening with the Hindi Mental State Examination (HMSE). The HMSE cutoff score on screening for dementia was $\leq 23 .{ }^{15,16}$ The diagnosis in each case was supported by magnetic resonance imaging of the brain. A characteristic pattern of medial temporal predominant cerebral atrophy was necessary for diagnosis of $A D$ cases, while ruling out other causes such as cerebrovascular disease. The healthy controls $(n=6)$ were randomly chosen from patients undergoing operative procedures under spinal anesthesia. All healthy controls underwent evaluation pre-operatively (HMSE and general questions about forgetfulness) to exclude dementia, and informed consent was taken from each, to include them in the study. The study was approved by the institutional ethics committee.

Patients of AD who had already been diagnosed previously and on medications were excluded. Patients with any severe acute illness requiring hospitalization or leading to delirium, any condition such as severe deafness, blindness or other illness which would interfere with neuropsychological testing were excluded from the study. In control group, those with chronic illnesses were excluded from the study.

2.4. Study parameters: The basic demographic data of each participant of the study, including HMSE scores were collected in pre-designed case report form.

2.5. Sample preparation and trypsin digestion in solution: Protein content in CSF samples was estimated by Bradford's reagent; then the samples were reduced and alkylated with $100 \mathrm{mM} \mathrm{DTT}$ at $56^{\circ} \mathrm{C}$ for $15 \mathrm{~min}$ and $200 \mathrm{mM}$ iodoacetamide in the dark for $15 \mathrm{~min}$, respectively. Further overnight digestion of the sample was carried out with trypsin in $1: 25$ ratio at $37^{\circ} \mathrm{C}$. The digested peptides were planned for LC-MS/MS analysis. ${ }^{17}$

2.6. Reverse phase liquid chromatography fractionation (RPLC): Peptide digest ( $3 \mu \mathrm{g})$ was separated by using Eksigent MicroLC 200 system (Eksigent, Dublin, CA) equipped with Eksigent C18-reverse phase column $(100 * 0.3 \mathrm{~mm}, 3 \mu \mathrm{m}, 120 \AA)$. The sample was loaded onto the column with $97 \%$ of mobile phase $A$ ( $100 \%$ water, $0.1 \%$ Formic acid (FA)) and $3 \%$ of mobile phase B ( $100 \%$ ACN (Acetonitrile), $0.1 \%$ FA) at 7 $\mu \mathrm{L} / \mathrm{min}$ flow rate. Peptides were eluted with a 120 min linear gradient of $3 \%$ to $50 \%$ mobile phase $B$.

\subsection{Tandem mass spectrometric analysis (LC-MS/MS): $3 \mu \mathrm{l}(\sim 1-2 \mu \mathrm{g})$ of peptide was loaded for each} sample in column for IDA analysis and then sample was run for 2 hours. Column was washed with $3 \%$ ACN. $1 \mu \mathrm{l}(\sim 1-2 \mu \mathrm{g})$ of peptide was loaded for each sample in column for SWATH analysis and then the sample was run for 2 hours. Column was washed with 3\% ACN. AB Sciex Triple TOF 5600 instrument and IDA (Information Dependent Acquisition) protocol was used for the construction of fragment ion library. The spectral alignment and targeted data extraction of SWATH-MS data was performed using Peakview software, Version 1.2.03 (AB SCIEX, MA, USA). The peptide data was used for quantification of peptides of CSF using Markerview software, Version 1.2.1.1 (AB Sciex, MA, USA). The peptides with a $p$ value $\leq$ 0.05 were considered significant with respect to differential expression in AD cases and controls (cutoff 1.5-fold for upregulated proteins; 0.75 -fold for down regulated proteins). The peptide data from CSF 
samples of both $\mathrm{AD}$ and control groups was then compared with complete human proteome data available online.

2.8 Bioinformatics Analysis: The proteins identified by LC-MS/MS which were differentially expressed in the cerebrospinal fluid of $A D$ patients with respect to healthy controls were taken up for bio-informatics analysis. Here we focused on the physical interaction network between those proteins that were reported in proteomic profiling of CSF (both down and upregulated). A network map was created using STRING (version 11.0). ${ }^{18,19}$ In initial STRING analysis, the confidence score was taken to be medium (0.4). After removing outlier proteins that were not involved in network formation with other proteins, and increasing confidence score to 0.9 , specific proteins were identified. Enrichment analysis was performed for the PPI network obtained and an attempt was made to identify patterns in the findings.

\section{Results}

3.1. Demographic profile of AD patients and controls: The case group had 6 patients ( 5 males, 1 female). The mean age of the AD patients was 68.5 years $( \pm 8.11)$. The control group had 6 subjects ( 4 males, 2 females). The mean age of the control group was 67.08 years $( \pm 5.31)$. The median duration of disease symptoms for case group was 2.5 years and the mean HMSE score was $17.5( \pm 3.93)$.

3.2. Proteomic analysis of CSF: We found 13 proteins to be upregulated in the CSF of AD patients as compared to controls. These included the proteins Contactin1 (CNTN1), Transthyretin (TTR), Apolipoprotein E (APOE), Gelsolin (GSN) and others (Figure 1). 30 proteins were found to be down regulated in the CSF of $A D$ patients and these included Prothrombin (F2), Apolipoprotein $\mathrm{H}(\mathrm{APOH})$, proenkephalin A (PENK), Neuronal pentraxin receptor (NPTXR), Alpha 2HS glycoprotein (AHSG) and others (Figure 2).

\subsection{Bioinformatics Analysis}

STRING (version 11.0): The protein-protein interaction (PPI) network of 13 upregulated and 30 downregulated proteins in CSF of AD patients is shown in Figure 3. 20 proteins were found to be participating in a closed network; others were outlier proteins which did not form part of the network. After removing these outliers, a simplified PPI network at higher confidence score of 0.9 was generated involving 10 proteins (Figure 4). These proteins are PENK (down regulated), F2 (down regulated), LGALS3BP (up regulated), AHSG (down regulated), APOE (up regulated), SAA1 (down regulated) SPP1 (downregulated), CD44 (down regulated), IGF (down regulated) and APOH (down regulated).

\section{Discussion}

The current study aimed to identify proteins which are significantly up and down regulated in the cerebrospinal fluid of AD patients with respect to controls. In the absence of similar work in the north Indian population, the study focused on both replicating previous findings from diverse experimental designs as also identifying tentative new biomarker proteins. LC-MS/MS analysis allowed us to identify 
13 upregulated and 30 down regulated proteins. Out of these, 10 were found to interact in a closed network at high confidence level.

\subsection{Up regulated Proteins:}

LGALS3BP (Galectin-3-binding protein) is a protein involved in integrin-mediated cell adhesion. (UniProt) LGALS3BP contains a scavenger receptor cysteine-rich (SRCR) domain which is associated with the innate immunity response. It also harbors a BTB/POZ domain which is involved in a wide variety of biologic processes including transcription regulation, cytoskeletal rearrangement, and protein ubiquitination. ${ }^{21}$ LGALS3BP has been found upregulated in serum of various diseases such as cancer and HIV. It is also seen that LGALS3BP is a main ligand for sialic acid-binding immunoglobulin-like lectins (Siglecs) that transmit inhibitory signals via intracellular immune receptor and modulate the immune response. ${ }^{22}$ iTRAQ labeling and tandem mass spectrometry based analysis on whole protein extracts of Alzheimer's brains identified LGALS3BP as one of the overexpressed polypeptides. ${ }^{23}$ Similar findings emerged from our study with significantly high levels in CSF of AD subjects as compared to controls. Dysregulation in immune response is one of the mechanisms involved in AD pathogenesis, and LGALS3BP may play a role in the process.

APOE (Apolipoprotein E) mediates lipid transport between organs including the central nervous system via the plasma and interstitial fluids. Apolipoprotein $\mathrm{E}$ gene $(A P O E)$ is the major risk gene identified as yet for late onset AD (LOAD) and has three major isoforms: E2, E3, E4. The APOE4 allele has been found to be significantly associated with $A D{ }^{24}$ APOE is involved in the production, conversion and clearance of plasma lipoproteins. APOE also plays an important role in regulation of neuronal survival and sprouting. ${ }^{25}$ APOE affects the clearance and aggregation of monomeric $A \beta$ in the brain of mice by modulating the microglial response. APOE deficient mice have shown a decrease in plaque-associated micro-gliosis and also decreased expression of activated microglial gene. ${ }^{26}$ Neurons fulfill their need for cholesterol by APOE mediated endocytosis of lipoprotein. Interference in the delivery of cholesterol is associated with neurodegeneration. ${ }^{27}$ In addition, immuno-histochemical analysis of Alzheimer's disease brains suggested APOE interacts with $\beta$-amyloid and influences its aggregation. ${ }^{28}$

\subsection{Down regulated Proteins:}

F2 (Prothrombin) is the precursor of thrombin that is known to be expressed in the brain and possesses growth modulating properties. F2 has been hypothesized to be involved in the pathogenesis of Alzheimer's disease (AD). ${ }^{29}$ Plasma circulating prothrombin is deposited within the microvessel wall of the brain and surrounding the neuropil due to thinning and discontinuities within the vascular basement membrane of the blood-brain barrier. This leakage is more common in AD patients with at least one APOE4 allele, and is directly proportional to the severity of disease. ${ }^{30}$ Thrombin has been detected immuno-histochemically in brain tissue of Alzheimer's disease (AD) patients and age-matched controls. There have been suggestions that thrombin formation from prothrombin probably takes place in the $A D$ brain. ${ }^{31}$ However, CSF F2 concentration has not been demonstrated to be altered in AD. ${ }^{29}$ In our study, we 
found significant down regulation of $F 2$ in the CSF of $A D$ patients. This could be a result of blood brain barrier leakage, prothrombin deposition in the microvasculature and conversion to thrombin.

PENK (Proenkephalin) is a precursor fragment of the neuropeptide hormone enkephalin which also has a role in blood brain barrier integrity. ${ }^{32}$ PENK is expressed in the nervous system ${ }^{33}$ and contributes to various physiologic functions including glial cell proliferation, responses to stress and aging. ${ }^{34}$ It is also a member of the opioid system that contributes to the hyper phosphorylation of tau. The dysfunction of the opioid system is linked to cholinergic neuronal degeneration and AD pathogenesis. ${ }^{35}$ In our study, we found significant down regulation of PENK which may be in line with opioid system dysfunction that happens in AD brains.

IGF2 (Insulin-like growth factor II) is a major fetal growth hormone in mammals which is also involved in tissue differentiation and glucose metabolism and acts as a ligand for integrin. ${ }^{36}$. Administration of recombinant IGF2 in the hippocampal region of aged wild-type mice enhances memory and promotes dendritic spine formation. ${ }^{37}$ Infusion of IGF2 in transgenic AD mice also replicated the same findings. IGF2 reduces the number of hippocampal $A \beta 40$ - and $A \beta 42$-positive amyloid plaques in transgenic mice model. IGF2 also increases the expression of proteins involved in neurogenesis including NGF, BDNF, NT3 and IGF1 and of doublecortin in the hippocampal region. A significantly decreased expression of IGF2 was found in the current study in the CSF of AD patients. This may point towards a failure of the neuroprotective mechanism during disease pathogenesis. The finding is at variance to a previous report of significantly elevated level of immunoreactive IGF2 in both serum and CSF of AD patient group. ${ }^{38}$

APOH (Beta-2-glycoprotein 1) is a lipid-binding protein that prevents activation of the intrinsic pathway of blood coagulation. Studies have suggested that APOH has links to cognitive aging and Alzheimer's disease. A study on the plasma of 664 subjects with mild cognitive impairment and normal cognition suggested the association of APOH with increased risk of cognitive decline in cognitively normal people and its potential as a biomarker. ${ }^{39} \mathrm{~A}$ multiplex immunoassay was utilized in CSF samples of moderate-tosevere AD subjects to analyze 90 proteins including APOH. Significant increase was found in APOH level only in those with moderate to severe $A D .{ }^{40}$ In our study we found a significant decrease in the expression of $\mathrm{APOH}$ in the CSF of AD subjects as compared to controls which differs from previous evidence. The reason for this is unclear.

SAA1 (Serum amyloid A-1 protein) and SAA2 (Serum amyloid A-2 protein) are major acute-phase reactant proteins. They play an important role in cell chemotaxis and cytokine-mediated signaling pathways. ${ }^{41,42}$ The exact mechanism how these affect neurodegeneration pathogenesis is less established but down regulation of SAA1 and SAA2 is seen in PD subjects. ${ }^{43}$ Potential involvement in tau hypo-phosphorylation is also seen in mice model. Lipopolysaccharides (LPS) induce the expression of SAA in the brain that leads to suppression of tau hyper phosphorylation by activating glial response. ${ }^{44}$ In the current study, we observed a down regulation of SAA1 and SAA2 proteins in the CSF of AD patients. Low SAA levels may result in tau hyperphosphorylation and the $A D$ pathogenetic cascade. 
AHSG (Alpha-2-HS-glycoprotein) also known as fetuin-A, is a highly glycosylated protein ${ }^{45}$ which is primarily secreted by the liver and promotes endocytosis and possesses opsonic properties. ${ }^{46} \mathrm{It}$ is a physiological inhibitor of insulin receptor tyrosine kinase. ${ }^{47}$ Studies have also suggested that AHSG has an important role in neurodegenerative diseases. A significant decrease in the expression of AHSG is reported from the cerebral cortex, hippocampus, and thalamus in transgenic mice model of $A D$ with chronic hypoperfusion. ${ }^{48}$ Our study found a decreased CSF level of AHSG in AD patients, a finding which concurred with previous observations. ${ }^{45}$

SPP1 (secreted phosphoprotein 1/ Osteopontin) is an extracellular matrix protein, involved in cellular migration, fusion, and motility which is biosynthesized by a variety of tissue types including the brain. ${ }^{49} \mathrm{~A}$ study by Comi et al revealed osteopontin (OPN) to be significantly increased in the CSF of AD patients and also higher in the early disease phases. ${ }^{50} \mathrm{~A}$ targeted proteomic multiple reaction monitoring (MRM)based assay was used by another group to measure multiple candidate CSF biomarkers in AD patients and showed significant elevation of osteopontin. ${ }^{51}$ The downregulation of SPP1 observed in the current study is unexplained and needs validation in large populations.

CD44 (CD44 antigen) is a cell-surface receptor that plays an important role in cell-cell interaction, cell adhesion, and migration. CD44 also co-ordinates calcium mobilization signaling and actin-mediated cytoskeleton reorganization which are essential for cell migration and adhesion. ${ }^{52}$ The expression of CD44 splice variants is significantly higher in postmortem hippocampal samples of AD patients compared to non-AD controls. It has hence been suggested that CD44 splice variants contribute to $A D$ pathology. ${ }^{53}$ Expression of CD44 is found predominantly in the white matter and also in both glial cells and neuronal cells of the human brain. ${ }^{54}$ In the grey matter, CD44 is expressed in astrocytes and it is found that expression of CD44 positive astrocytes increases dramatically in the AD brain. It serves as a receptor for various proteins like hyaluronic acid, collagen types I and VI. ${ }^{55}$ Increased expression of CD44 is also reported in the lymphocytes derived from $A D$ subjects with respect to age-matched controls. ${ }^{56}$ In our study we found significant down regulation of CD44 in CSF. This finding needs further validation in larger sample sizes.

\subsection{Tentative mechanisms in pathogenesis of AD-pointers from STRING analysis}

The proteins found to be differentially expressed in the CSF of AD patients and involved in a closed PPI network were analyzed for functional enrichment in STRING. With respect to biological processes, the largest enrichment effect (strength $>2$ ) was observed for regulation of behavioral fear response (APOE and PENK), followed by negative regulation of fibrinolysis (APOH and F2), locomotory exploration behavior (APOE and PENK), intrinsic pathway of blood coagulation (APOH and F2), negative regulation of platelet activation (APOE and F2), the steroid catabolic process (APOE and SPP1), acute phase response ( $F 2, A H S G$ and SAA1), the response to vitamin D (PENK and SPP1), negative regulation of blood coagulation (APOE, APOH and F2), and negative regulation of endothelial cell proliferation (APOE and $\mathrm{APOH}$ ) (Figure 5). We observed that APOE, APOH, F2 and PENK are involved in multiple of these 
biological processes. The interactions of each of PENK, F2, SPP1 and APOH with APOE, a known pathogenetic marker of $A D$ may be of relevance, indicating putative involvement.

Among the local STRING network clusters, 6 clusters were significantly enriched. One that we found to be particularly relevant was regulation of insulin like growth factor transport and uptake which was a component of 3 out of the 6 clusters, and included AHSG, APOE, IGF2 and F2 (Figure 6). Insulin interacts with amyloid- $\beta$ precursor protein (AßPP or APP) although the exact mechanisms remain unclear. ${ }^{57}$ Insulin resistance in brain has been demonstrated to correlate with AD pathogenesis. ${ }^{58}$ IGF-2 binds and activates insulin receptors and regulates neurotransmitter release, memory consolidation and neuroprotection. ${ }^{59}$ IGF-2 triggers its signaling processes through MAPK and PI3K/Akt signaling pathways. ${ }^{60}$ Extensive abnormalities in IGF-2 signaling mechanisms leads to reduced levels of insulin receptor substrate (IRS) mRNA and PI3K/Akt. This causes an increase in glycogen synthase kinase-3 $\beta$ activity that decreases glycogen synthesis and increases amyloid precursor protein mRNA expression. ${ }^{61}$ Decrease in IGF2 levels may have a decremental effect on its signaling pathways. Prothrombin proteolyzes the microtubuleassociated protein tau. ${ }^{62}$ Lowering of prothrombin maybe an indirect marker of greater conversion to thrombin. The binding of thrombin to its receptor stimulates inflammatory cytokines including IL- 6 and monocyte chemoattractant protein-1 (MCP-1) which leads to insulin resistance by inactivating IRS/Akt signaling pathway. ${ }^{63} \mathrm{AHSG}$ also acts on the insulin-signaling pathway. It may be an alternative marker for insulin resistance. ${ }^{64}$ It has been shown that the serum concentration of AHSG is significantly elevated in diet-induced obese mice and it inhibits the metabolic effects of insulin. ${ }^{65}$ AHSG inhibits insulinstimulated insulin receptor autophosphorylation, insulin-induced tyrosine phosphorylation of insulin receptor substrate-1 (IRS1). ${ }^{66}$ Evidence currently is lacking for the role of CSF AHSG in brain limited insulin resistance. Insulin binding to its receptor in the nucleus accumbens (NAC) of the brain has been shown to activate the opioid receptor-mediated release of presynaptic glutamate which influences cognition and the decision-making processes. ${ }^{67}$ The involvement of proenkephalin in similar interactions of the insulin and opioid pathways may be hypothesized. There is a already a large evidence pool for the link between APOE and cerebral insulin. ${ }^{68}$

As regards to molecular function, a process of interest for us was glycosaminoglycan (GAG) binding. Five proteins were annotated with GAG binding (APOE, APOH, F2, SAA1, and CD44) (Figure 7). GAGs are the major components of extracellular matrix which participate in tissue homeostasis and self-healing. ${ }^{69}$ In Alzheimer's disease pathogenesis, $A \beta$ plaques bind to different extracellular membrane components including GAGs. GAGs enhance the formation of $A \beta$ fibrils that lead to its extracellular accumulation. ${ }^{70}$ The C-terminal domain of APOE binds with GAGs present on the cell surface. APOE4 has a greater ability to bind with GAGs which is physiologically significant. ${ }^{71}$ It is observed that GAGs along with acetaldehyde directly affects $\mathrm{F} 2$ to mediate the anticoagulant reaction. ${ }^{72}$ Acetaldehyde- a product of ethanol metabolism is involved in the cross linking of F2 and GAGs; how this anticoagulant property of GAGs is important in AD pathogenesis is not established yet. Surface plasmon resonance spectroscopy and molecular modeling based analysis have shown that cell surface HS (heparin sulphate) converts 
HDL-associated SAA into AA amyloid fibrils in cell culture. ${ }^{73} \mathrm{~A}$ similar interaction may be a supportive event in causing accumulation of amyloid proteins in the brain. To mediate cell adhesion and intracellular signaling, CD44 binds with the chondroitin sulfate (CS) proteoglycan of GAGs. ${ }^{74} \mathrm{CS}$ proteoglycan is critical in spinal injury and diseases of the central nervous system. They play an inhibitory effect on glial scar formation and improve the regeneration and functional recovery of neurons. ${ }^{75} \mathrm{CD} 44$ is also a major receptor for hyaluronan ( $\mathrm{HA})$ which is a remarkably versatile GAG involved in cell signaling and inflammatory pathologies.

\section{Conclusion}

Multiple studies have been performed on cerebrospinal fluid proteomics in Alzheimer's disease patients. However, findings from different geographical zones are needed to support previous data and lend new insights into the pathogenesis of AD. Although our study had a small sample size, we tried to include a broader group of proteins in bioinformatic analysis by using thresholds of 1.5 and 0.75 in fold-change. The identification of F2 and PENK as tentative AD biomarkers and their close interaction with APOE is of interest. Further, the role of the insulin regulatory pathway and glycosaminoglycan binding in $A D$ pathogenesis need to be explored in future studies. Utilizing STRING for the creation of protein-protein interaction networks is an attractive concept for proteomic analysis of tandem mass spectrometric data. The findings from our study would need validation in larger groups of patients.

\section{Declarations}

\section{Funding:}

a. National Programme for Health Care of the Elderly, Ministry of Health and Family Welfare, Government of India.

b. Indian Council of Medical Research - Senior Research Fellowship grant 3/1/2/(23)/GER/2019-NCDII

\section{Conflicts of interest/Competing interests: None}

Availability of data and material: On Request

\section{Code availability: Not applicable}

Authors' contributions: AM wrote the article and performed all experimental work; MSB and YRP assisted in experimental work; ISG guided planning of experiment and assisted in critical analysis of findings; SSC planned the experiments and bioinformatic analysis and edited the final version of manuscript.

Ethics approval: Study was approved by the institutional ethics committee of Institute of Medical Sciences, Banaras Hindu University, Varanasi. 
Consent to participate: Written and informed consent was taken from each participant (Alzheimer's disease patient or control) to include them in the study. For participants unable to provide consent, consent was taken from legal guardian.

Consent for publication: Consent to publish was taken

ACKNOWLEDGMENTS- None

\section{References}

1. Wilson, R. S. et al. The natural history of cognitive decline in Alzheimer's disease. Psychol. Aging 27, 1008 (2012).

2. Chandra, V. et al. Incidence of Alzheimer's disease in a rural community in India: the Indo-US study. Neurology 57, 985-989 (2001).

3. Ho, L., Fivecoat, H., Wang, J. \& Pasinetti, G. M. Alzheimer's disease biomarker discovery in symptomatic and asymptomatic patients: experimental approaches and future clinical applications. Exp. Gerontol. 45, 15-22 (2010).

4. Karlawish, J., Jack, C. R., Rocca, W. A., Snyder, H. M. \& Carrillo, M. C. Alzheimer's disease: The next frontier-Special Report 2017. Alzheimer's Dement. 13, 374-380 (2017).

5. Wang, B., Canestaro, W. J. \& Choudhry, N. K. Clinical evidence supporting pharmacogenomic biomarker testing provided in US Food and Drug Administration drug labels. JAMA Intern. Med. 174, 1938-1944 (2014).

6. Chakrabarti, S. et al. Metabolic risk factors of sporadic Alzheimer's disease: implications in the pathology, pathogenesis and treatment. Aging Dis. 6, 282 (2015).

7. Thambisetty, M. et al. Association of plasma clusterin concentration with severity, pathology, and progression in Alzheimer disease. Arch. Gen. Psychiatry 67, 739-748 (2010).

8. Becker, M., Schindler, J. \& Nothwang, H. G. Neuroproteomics-the tasks lying ahead. Electrophoresis 27, 2819-2829 (2006).

9. Choe, L. et al. 8-plex quantitation of changes in cerebrospinal fluid protein expression in subjects undergoing intravenous immunoglobulin treatment for Alzheimer's disease. Proteomics 7, 36513660 (2007).

10. Henkel, A. W. et al. Multidimensional plasma protein separation technique for identification of potential Alzheimer's disease plasma biomarkers: a pilot study. J. Neural Transm. 119, 779-788 (2012).

11. Ringman, J. M. et al. Proteomic changes in cerebrospinal fluid of presymptomatic and affected persons carrying familial Alzheimer disease mutations. Arch. Neurol. 69, 96-104 (2012).

12. Kang, S. et al. PiB-PET imaging-based serum proteome profiles predict mild cognitive impairment and Alzheimer's disease. J. Alzheimer's Dis. 53, 1563-1576 (2016). 
13. Dayon, L. et al. Alzheimer disease pathology and the cerebrospinal fluid proteome. Alzheimers. Res. Ther. 10, 66 (2018).

14. Sathe, G. et al. Quantitative proteomic profiling of cerebrospinal fluid to identify candidate biomarkers for Alzheimer's disease. PROTEOMICS-Clinical Appl. 1800105 (2018).

15. Tsolaki, M. et al. Hindi Mental State Examination (HMSE) as a screening test for illiterate demented patients. Int. J. Geriatr. Psychiatry 15, 662-664 (2000).

16. Ganguli, M. et al. A Hindi version of the MMSE: the development of a cognitive screening instrument for a largely illiterate rural elderly population in India. Int. J. Geriatr. Psychiatry 10, 367-377 (1995).

17. Suttapitugsakul, S., Xiao, H., Smeekens, J. \& Wu, R. Evaluation and optimization of reduction and alkylation methods to maximize peptide identification with MS-based proteomics. Mol. Biosyst. 13, 2574-2582 (2017).

18. Szklarczyk, D. et al. The STRING database in 2017: quality-controlled protein-protein association networks, made broadly accessible. Nucleic Acids Res. gkw937 (2016).

19. Schwartz, A. S., Yu, J., Gardenour, K. R., Finley Jr, R. L. \& Ideker, T. Cost-effective strategies for completing the interactome. Nat. Methods 6, 55-61 (2009).

20. UniProt, O. Galectin-3-binding protein (LGALS3BP).

21. Loimaranta, V., Hepojoki, J., Laaksoaho, O. \& Pulliainen, A. T. Galectin-3-binding protein: A multitask glycoprotein with innate immunity functions in viral and bacterial infections. J. Leukoc. Biol. 104, 777-786 (2018).

22. Läubli, H. et al. Lectin galactoside-binding soluble 3 binding protein (LGALS3BP) is a tumorassociated immunomodulatory ligand for CD33-related Siglecs. J. Biol. Chem. 289, 33481-33491 (2014).

23. Minjarez, B. et al. Identification of proteins that are differentially expressed in brains with Alzheimer's disease using iTRAQ labeling and tandem mass spectrometry. J. Proteomics 139, 103-121 (2016).

24. Misra, A., Shubhra Chakrabarti, S., Singh Gambhir, I., Kaur, U. \& Prasad, S. APOE4 allele in north Indian elderly patients with dementia or late onset depression-a multiple-disease case control study. Mol. Biol. Res. Commun. 8, 135-140 (2019).

25. UniProt, O. Apolipoprotein E (APOE).

26. Fenkçi IV, Maternal Fizyoloji. 'Çiçek MN, Ed.' Kadın Hastalıkları ve Doğum Bilgisi, Öncü Basımevi, A. (2004): 161-9. Scholar (4). (4AD). doi:10.1163/_q3_SIM_00374

27. Pfrieger, F. W. Cholesterol homeostasis and function in neurons of the central nervous system. Cell. Mol. Life Sci. C. 60, 1158-1171 (2003).

28. Namba, Y., Tomonaga, M., Kawasaki, H., Otomo, E. \& Ikeda, K. Apolipoprotein E immunoreactivity in cerebral amyloid deposits and neurofibrillary tangles in Alzheimer's disease and kuru plaque amyloid in Creutzfeldt-Jakob disease. Brain Res. 541, 163-166 (1991).

29. Lewczuk, P. et al. Prothrombin concentration in the cerebrospinal fluid is not altered in Alzheimer's disease. Neurochem. Res. 24, 1531-1534 (1999). 
30. Zipser, B. D. et al. Microvascular injury and blood-brain barrier leakage in Alzheimer's disease. Neurobiol. Aging 28, 977-986 (2007).

31. Akiyama, H., Ikeda, K., Kondo, H. \& McGeer, P. L. Thrombin accumulation in brains of patients with Alzheimer's disease. Neurosci. Lett. 146, 152-154 (1992).

32. Hurlbut, D. E., Evans, C. J., Barchas, J. D. \& Leslie, F. M. Pharmacological properties of a proenkephalin A-derived opioid peptide: BAM 18. Eur. J. Pharmacol. 138, 359-366 (1987).

33. Squillario, M. \& Barla, A. A computational procedure for functional characterization of potential marker genes from molecular data: Alzheimer's as a case study. BMC Med. Genomics 4, 55 (2011).

34. UniProt, O. Proenkephalin(PENK).

35. Cai, Z. \& Ratka, A. Opioid system and Alzheimer's disease. Neuromolecular Med. 14, 91-111 (2012).

36. UniProt, O. Insulin-like growth factor II (IGF2).

37. Pascual-Lucas, M. et al. Insulin-like growth factor 2 reverses memory and synaptic deficits in APP transgenic mice. EMBO Mol. Med. 6, 1246-1262 (2014).

38. Suh, H.-S., Zhao, M.-L., Derico, L., Choi, N. \& Lee, S. C. Insulin-like growth factor 1 and 2 (IGF1, IGF2) expression in human microglia: differential regulation by inflammatory mediators. $J$. Neuroinflammation 10, 805 (2013).

39. Song, F. et al. Plasma apolipoprotein levels are associated with cognitive status and decline in a community cohort of older individuals. PLoS One 7, e34078 (2012).

40. Öhrfelt, A. et al. Screening for new biomarkers for subcortical vascular dementia and Alzheimer's disease. Dement. Geriatr. Cogn. Dis. Extra 1, 31-42 (2011).

41. UniProt, O. SAA1 (Serum amyloid A-1 protein (SAA1).

42. UniProt, O. Serum amyloid A-2 protein (SAA2).

43. Planken, A. et al. Looking beyond the brain to improve the pathogenic understanding of Parkinson's disease: implications of whole transcriptome profiling of Patients' skin. BMC Neurol. 17, 6 (2017).

44. Liu, J., Wang, D., Li, S.-Q., Yu, Y. \& Richard, D. Y. Suppression of LPS-induced tau hyperphosphorylation by serum amyloid A. J. Neuroinflammation 13, 28 (2016).

45. Geroldi, D. et al. Genetic association of alpha2-Heremans-Schmid glycoprotein polymorphism with late-onset Alzheimer's disease in Italians. Neurosci. Lett. 386, 176-178 (2005).

46. UniProt, O. Alpha-2-HS-glycoprotein (AHSG).

47. Dabrowska, A. M., Tarach, J. S., Wojtysiak-Duma, B. \& Duma, D. Fetuin-A (AHSG) and its usefulness in clinical practice. Review of the literature. Biomed Pap Med Fac Univ Palacky Olomouc Czech Repub 159, 352-359 (2015).

48. Shi, X. et al. Acute Anti-Inflammatory Markers ITIH4 and AHSG in Mice Brain of a Novel Alzheimer's Disease Model. J. Alzheimer's Dis. 1-9 (2019).

49. Marcondes, M. C. G. et al. Osteopontin expression in the brain triggers localized inflammation and cell death when immune cells are activated by pertussis toxin. Mediators Inflamm. 2014, (2014). 
50. Comi, C. et al. Osteopontin is increased in the cerebrospinal fluid of patients with Alzheimer's disease and its levels correlate with cognitive decline. J. Alzheimer's Dis. 19, 1143-1148 (2010).

51. Paterson, R. W. et al. A targeted proteomic multiplex CSF assay identifies increased malate dehydrogenase and other neurodegenerative biomarkers in individuals with Alzheimer's disease pathology. Transl. Psychiatry 6, e952 (2016).

52. UniProt, O. CD44 antigen(CD44).

53. Pinner, E. et al. CD44 Splice Variants as Potential Players in Alzheimer's disease Pathology. J. Alzheimer's Dis. 58, 1137-1149 (2017).

54. Dzwonek, J. \& Wilczynski, G. M. CD44: molecular interactions, signaling and functions in the nervous system. Front. Cell. Neurosci. 9, 175 (2015).

55. Akiyama, H., Tooyama, I., Kawamata, T., Ikeda, K. \& McGeer, P. L. Morphological diversities of CD44 positive astrocytes in the cerebral cortex of normal subjects and patients with Alzheimer's disease. Brain Res. 632, 249-259 (1993).

56. Uberti, D. et al. Increased CD44 gene expression in lymphocytes derived from Alzheimer disease patients. Neurodegener. Dis. 7, 143-147 (2010).

57. Shieh, J. C.-C., Huang, P.-T. \& Lin, Y.-F. Alzheimer's disease and diabetes: insulin signaling as the bridge linking two pathologies. Mol. Neurobiol. 57, 1966-1977 (2020).

58. Watson, G. S. \& Craft, S. The role of insulin resistance in the pathogenesis of Alzheimer's disease. CNS Drugs 17, 27-45 (2003).

59. Lara Fernández, E. et al. IGF-II AS A NEUROPROTECTIVE AND NEUROPLASTIC FACTOR IN AN OXIDATIVE DAMAGE MODEL INDUCED BY GLUCOCORTICOIDS. (2018).

60. Codina, M. et al. Metabolic and mitogenic effects of IGF-II in rainbow trout (Oncorhynchus mykiss) myocytes in culture and the role of IGF-II in the PI3K/Akt and MAPK signalling pathways. Gen. Comp. Endocrinol. 157, 116-124 (2008).

61. Steen, E. et al. Impaired insulin and insulin-like growth factor expression and signaling mechanisms in Alzheimer's disease-is this type 3 diabetes? J. Alzheimer's Dis. 7, 63-80 (2005).

62. Arai, T., Miklossy, J., Klegeris, A., Guo, J.-P. \& McGeer, P. L. Thrombin and prothrombin are expressed by neurons and glial cells and accumulate in neurofibrillary tangles in Alzheimer disease brain. $J$. Neuropathol. Exp. Neurol. 65, 19-25 (2006).

63. Mihara, M. et al. Inhibition of thrombin action ameliorates insulin resistance in type 2 diabetic $\mathrm{db} / \mathrm{db}$ mice. Endocrinology 151, 513-519 (2010).

64. Shim, Y. S. et al. Fetuin-A as an alternative marker for insulin resistance and cardiovascular risk in prepubertal children. J. Atheroscler. Thromb. 38323 (2017).

65. Ren, G. et al. Phosphorylation status of fetuin-A is critical for inhibition of insulin action and is correlated with obesity and insulin resistance. Am. J. Physiol. Metab. 317, E250-E260 (2019).

66. Andersen, G. et al. AHSG tag single nucleotide polymorphisms associate with type 2 diabetes and dyslipidemia: studies of metabolic traits in 7,683 white Danish subjects. Diabetes $\mathbf{5 7 , 1 4 2 7 - 1 4 3 2}$ 
(2008).

67. Oginsky, M. F., Santana-Rodriguez, Z. \& Ferrario, C. R. Insulin enhances presynaptic glutamate release in the nucleus accumbens via opioid receptor-mediated disinhibition. BioRxiv 517797 (2019).

68. Rhea, E. M., Raber, J. \& Banks, W. A. ApoE and cerebral insulin: Trafficking, receptors, and resistance. Neurobiol. Dis. 137, 104755 (2020).

69. Huynh, M. B. et al. Glycosaminoglycans from Alzheimer's disease hippocampus have altered capacities to bind and regulate growth factors activities and to bind tau. PLoS One 14, e0209573 (2019).

70. Ariga, T., Miyatake, T. \& Yu, R. K. Role of proteoglycans and glycosaminoglycans in the pathogenesis of Alzheimer's disease and related disorders: Amyloidogenesis and therapeutic strategies-a review. J. Neurosci. Res. 88, 2303-2315 (2010).

71. Yamauchi, Y. et al. Role of the $\mathrm{N}$-and $\mathrm{C}$-terminal domains in binding of apolipoprotein $\mathrm{E}$ isoforms to heparan sulfate and dermatan sulfate: a surface plasmon resonance study. Biochemistry 47, 67026710 (2008).

72. Brecher, A. S. \& Adamu, M. T. The effect of glycosaminoglycans with acetaldehyde on the activation of prothrombin. Can. J. Physiol. Pharmacol. 83, 431-438 (2005).

73. Noborn, F., Ancsin, J. B., Ubhayasekera, W., Kisilevsky, R. \& Li, J.-P. Heparan sulfate dissociates serum amyloid A (SAA) from acute-phase high-density lipoprotein, promoting SAA aggregation. J. Biol. Chem. 287, 25669-25677 (2012).

74. Fujimoto, T. et al. CD44 binds a chondroitin sulfate proteoglycan, aggrecan. Int. Immunol. 13, 359366 (2001).

75. Galtrey, C. M. \& Fawcett, J. W. The role of chondroitin sulfate proteoglycans in regeneration and plasticity in the central nervous system. Brain Res. Rev. 54, 1-18 (2007).

\section{Figures}




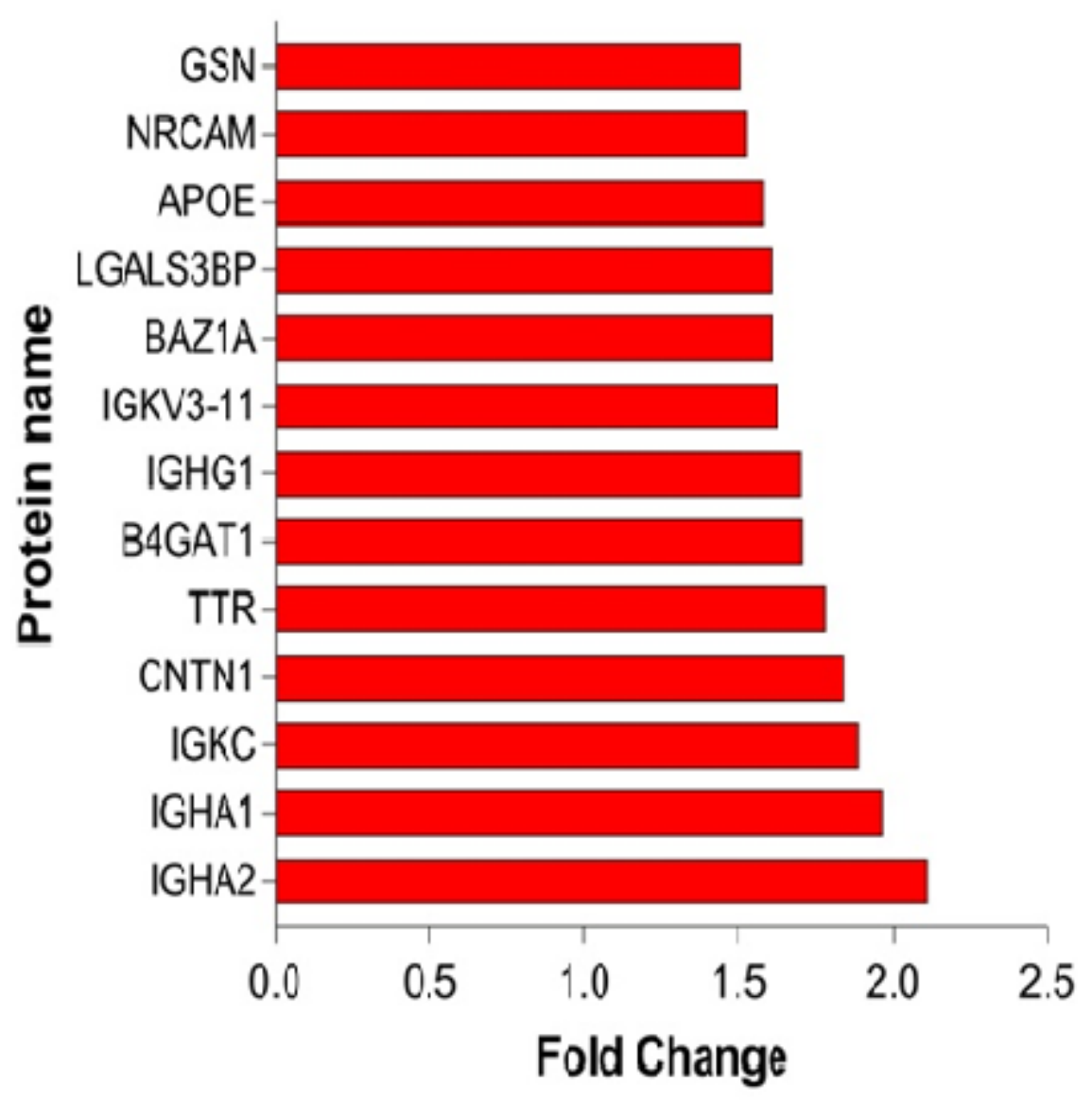

Figure 1

A graph of up regulated 13 proteins in CSF of AD patients as compared to controls (analyzed by GraphPad PRISM 7.00.159 Tool). Fold change $>1.5$ is considered as threshold level for up regulation. (GSN, Gelsolin; NRCAM, Neuronal Cell Adhesion Molecule; APOE, Apolipoprotein E; LGALS3BP, Galectin-3binding protein; BAZ1A, Bromodomain adjacent to zinc finger domain protein 1A; IGKV3-11, Immunoglobulin kappa variable 3-11; IGHG1, Immunoglobulin heavy constant gamma 1; B4GAT1, Beta1,4-glucuronyltransferase 1; TTR, Transthyretin; CNTN1, Contactin-1; IGKC, Immunoglobulin Kappa 
Constant; IGHA1, Immunoglobulin Heavy Constant Alpha 1; IGHA2, Immunoglobulin Heavy Constant Alpha 2;)

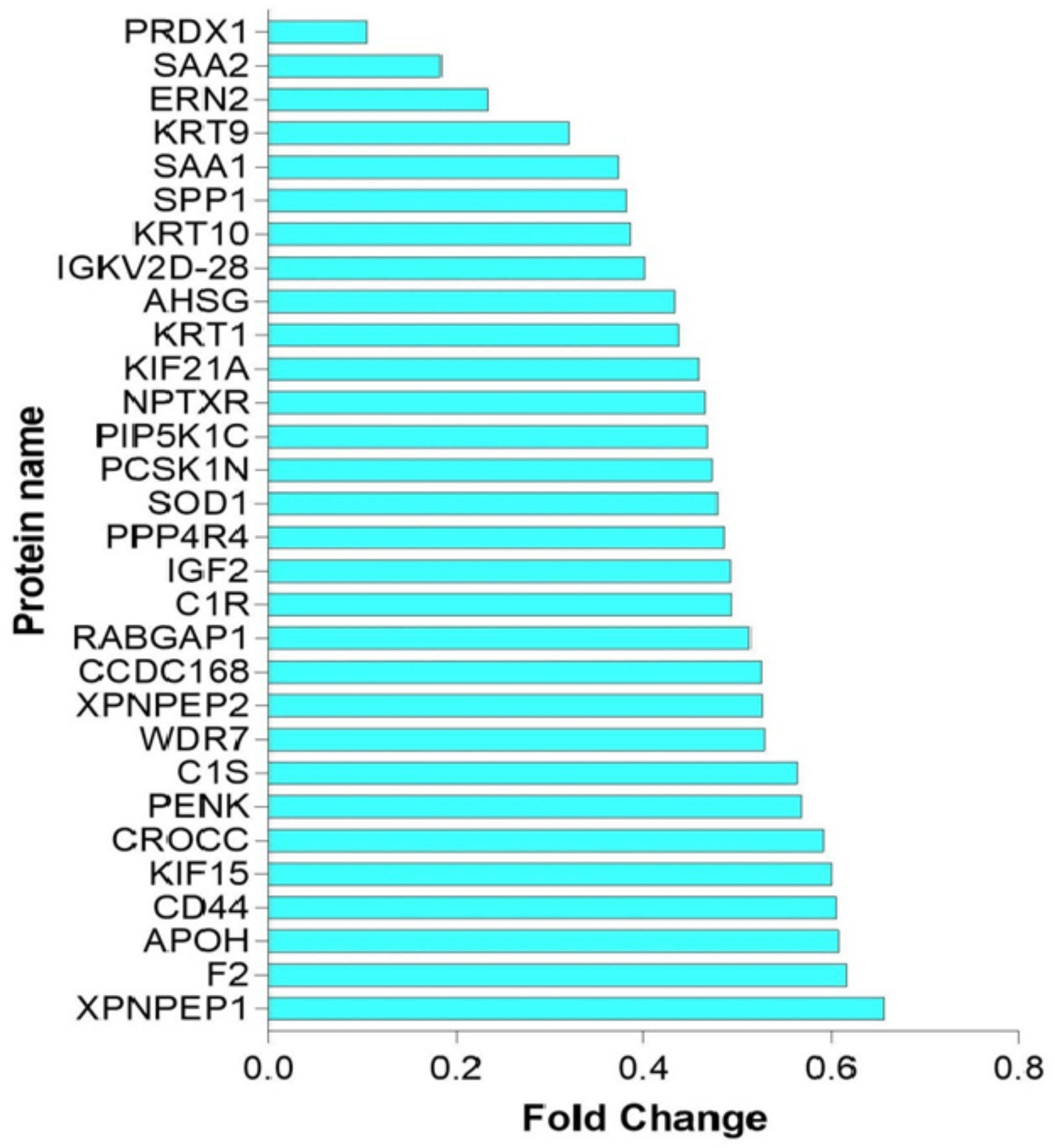

Figure 2

A graph of down regulated 30 proteins in CSF of AD patients as compared to controls (analyzed by GraphPad PRISM 7.00.159 Tool). Fold change $<0.75$ is considered as threshold level for down regulation. (PRDX1, Peroxiredoxin-1; SOD1, Superoxide dismutase [Cu-Zn]; SPP1, secreted phosphoprotein 1/ Osteopontin; CD44, CD44 antigen; AHSG, Alpha-2-HS-glycoprotein; KIF21A, Kinesin-like protein KIF21A; 
KIF15, Kinesin-like protein KIF15; PIP5K1C, Phosphatidylinositol 4-phosphate 5-kinase type-1 gamma; SAA1, Serum amyloid A-1 protein; SAA2, Serum amyloid A-2 protein; KRT1, type II cytoskeletal 1; KRT9, Keratin type I cytoskeletal 9; KRT10, Keratin type I cytoskeletal 10; C1R, Complement C1r subcomponent; C1S, Complement C1s subcomponent; IGKV2D-28, Immunoglobulin kappa variable 2D-28; ERN2, endoribonuclease IRE2; NPTXR, Neuronal pentraxin receptor; CCDC168, Coiled-coil domain-containing protein 168; PCSK1N, ProSAAS; F2, Prothrombin; PENK, Proenkephalin-A; PPP4R4, Serine/threonineprotein phosphatase 4 regulatory subunits 4 ; APOH, Beta-2-glycoprotein 1; IGF2, Insulin-like growth factor II; WDR7, WD repeat-containing protein 7; RABGAP1L, Rab GTPase-activating protein 1-like; CROCC, Rootletin; XPNPEP1, Xaa-Pro aminopeptidase 1; XPNPEP2, Xaa-Pro aminopeptidase 2)

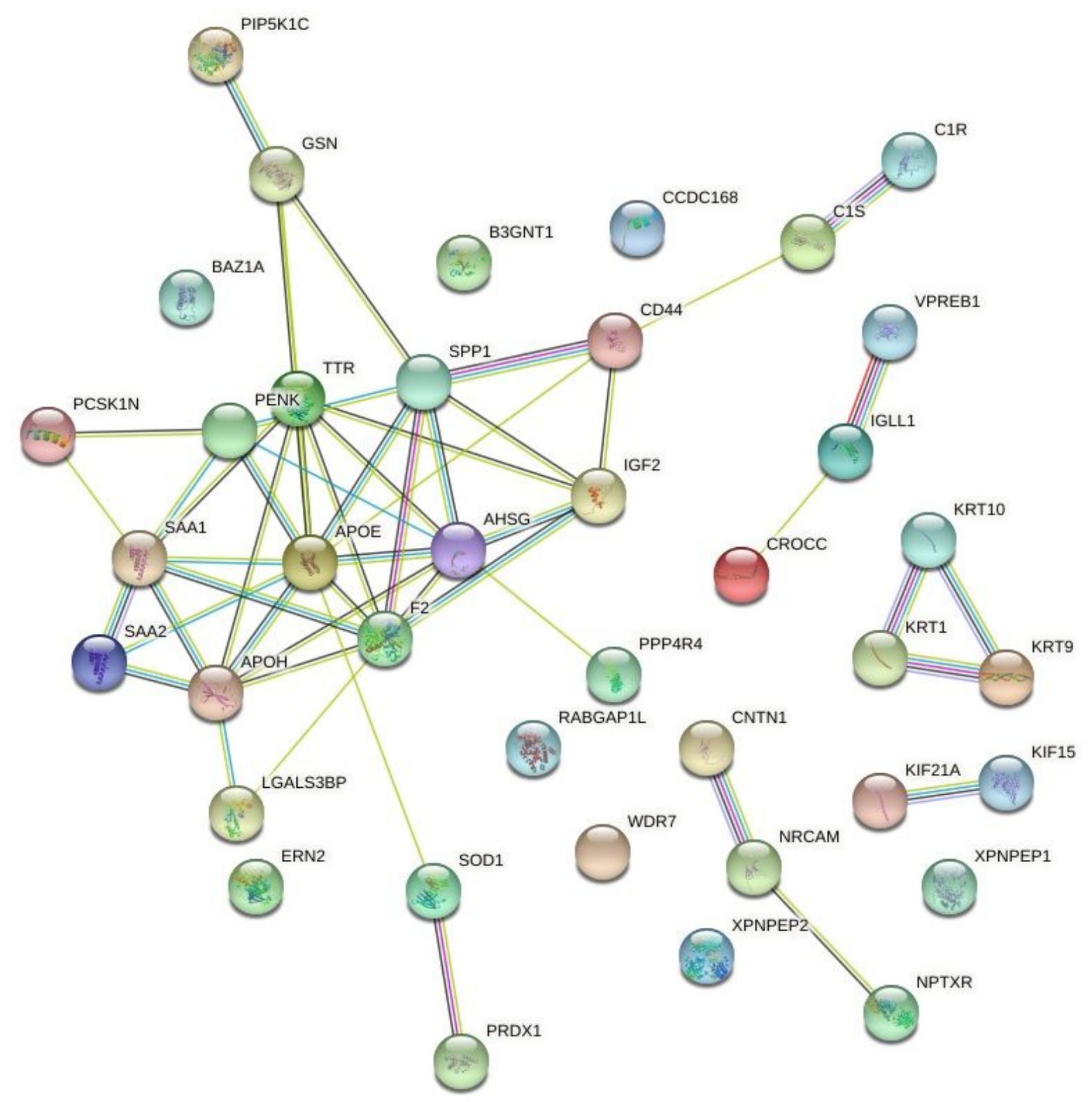

\section{Figure 3}

Protein-protein interaction (PPI) network of upregulated and downregulated proteins in the cerebrospinal fluid of AD patients (STRING 11.0; confidence level 0.40) 


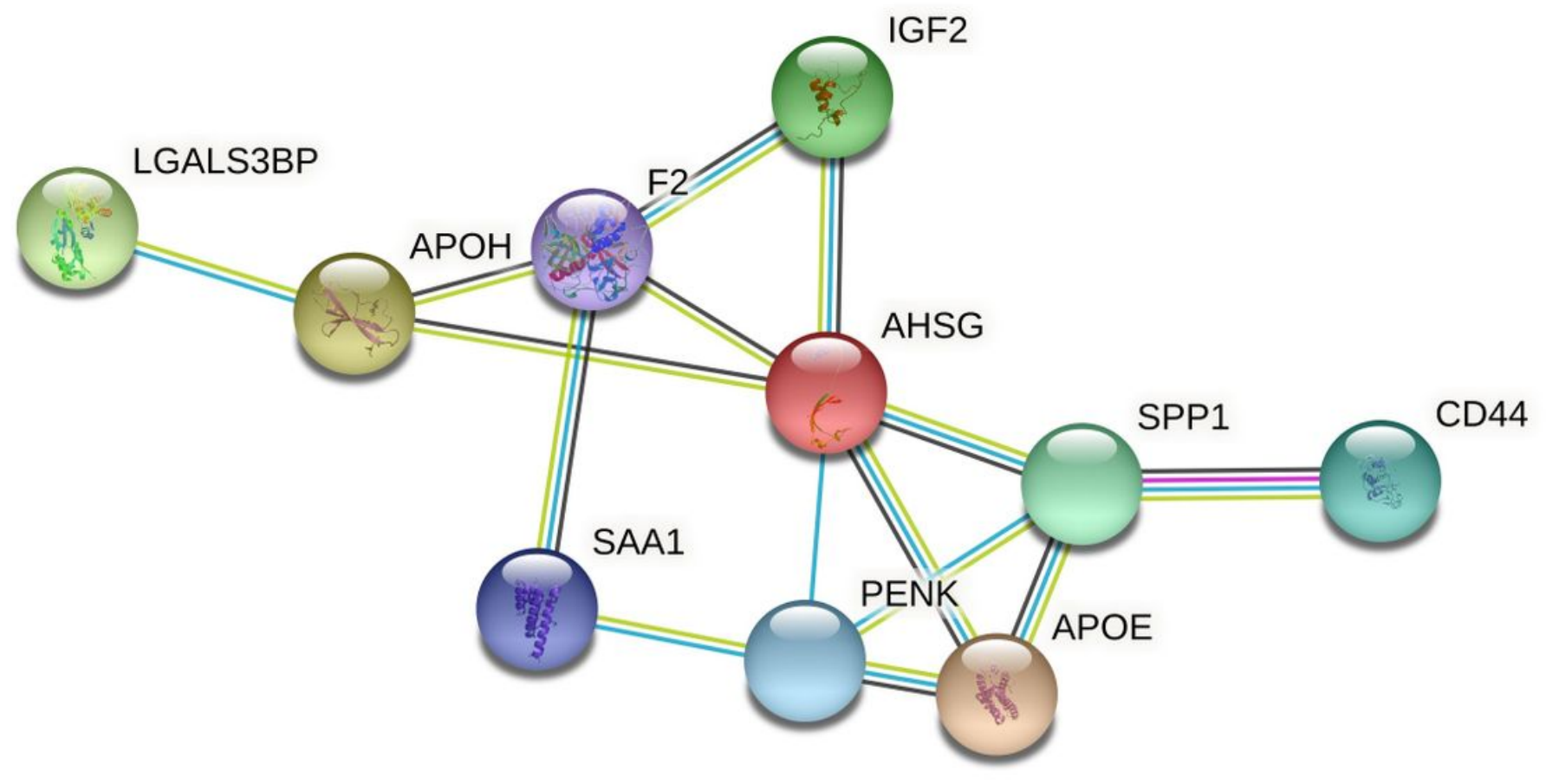

Figure 4

Simplified PPI network after removing outliers (STRING 11.0; confidence level 0.90). A network of 10 closely interacting proteins was obtained: PENK, F2, APOH, AHSG, SAA1, SPP1, CD44, IGF2 are downregulated proteins while LGALS3BP and APOE are upregulated.

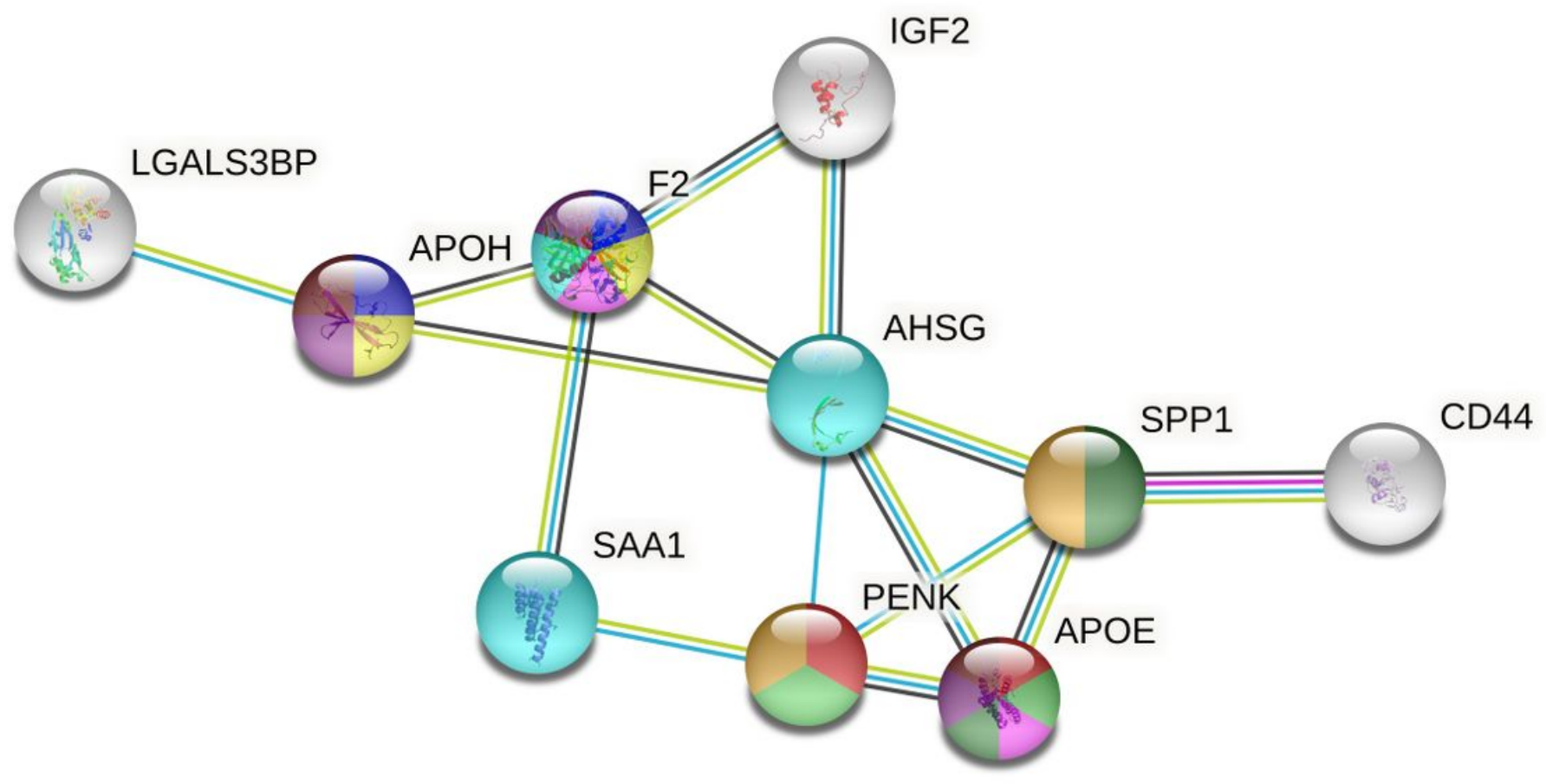

Figure 5 
Enrichment analysis of STRING PPI network-Biological process. Proteins which are annotated with a particular process have been color coded accordingly. Red- Regulation of behavioral fear response, Deep blue- Negative regulation of fibrinolysis, Light green- Locomotory exploration behavior, Yellow- Blood coagulation, intrinsic pathway, Pink- Negative regulation of platelets activation, Dark green- Steroids catabolic process, Light blue- Acute-phase response, Amber- Response to vitamin D, Violet- Negative regulation of blood coagulation, Brown- Negative regulation of endothelial cell proliferation.

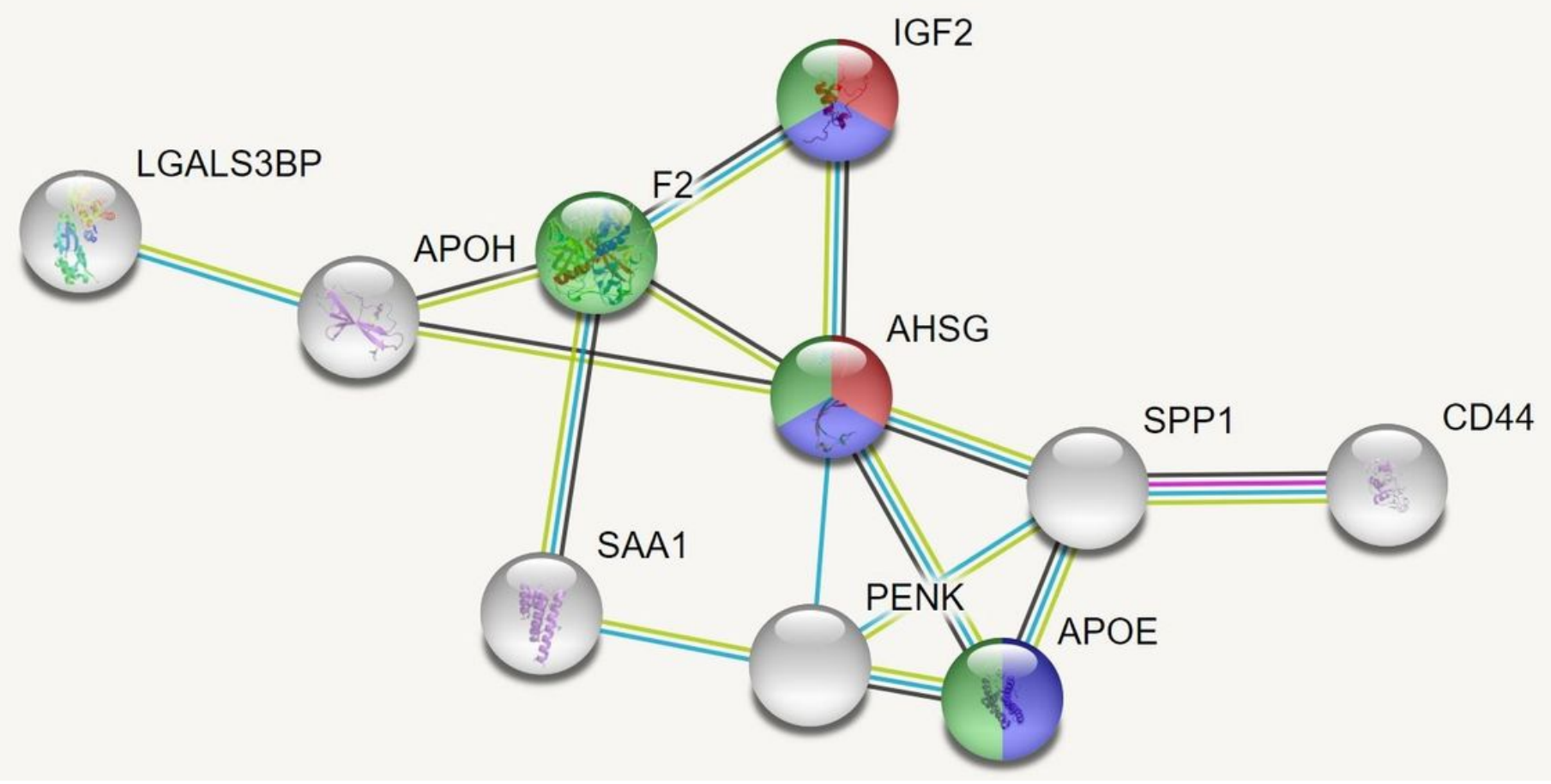

Figure 6

Enrichment analysis of STRING PPI network- Local network clusters. Proteins which are part of a particular cluster have been color coded accordingly. Red- Regulation of Insulin-like growth factor (IGF) transport and uptake by Insulin like growth factor binding protein (IGFBPs), Deep blue- Regulation of Insulin-like growth factor (IGF) transport and uptake by Insulin like growth factor binding protein (IGFBPs) and plasma lipoprotein particle, Light green- Regulation of Insulin-like growth factor (IGF) transport and uptake by Insulin like growth factor binding protein (IGFBPs) and formation of fibrin clot (clotting Cascade) 


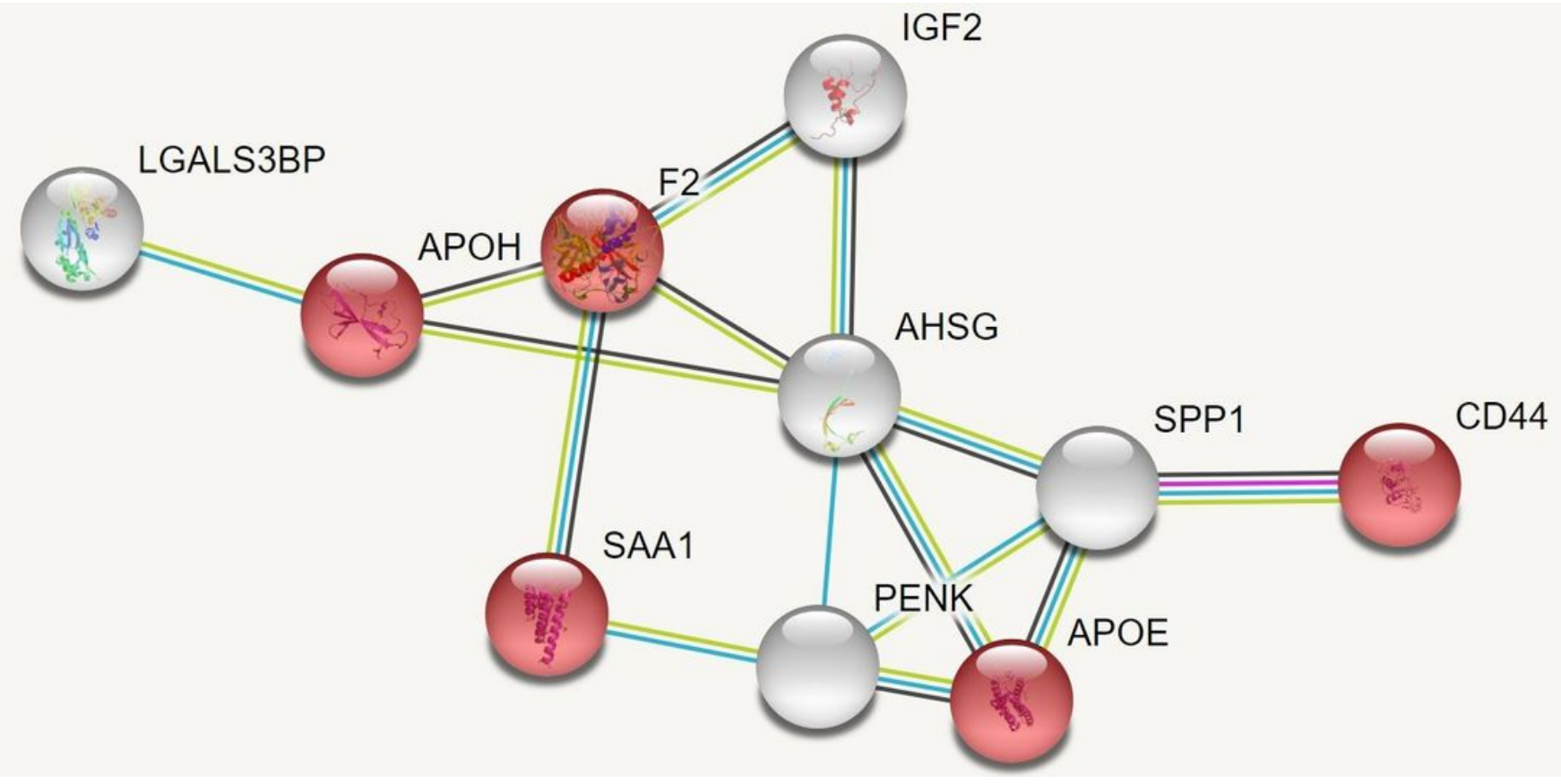

Figure 7

Enrichment analysis of STRING PPI network- Molecular function. Proteins involved in glycosaminoglycan binding have been labeled with red. 